\title{
Caracterización socioecológica de unidades familiares agroecológicas, con énfasis en la producción de leche
}

\author{
Socio-ecological characterization of agroecological family units, \\ with emphasis on milk production
}

Ana Paula Neves ${ }^{1 *}$, Leonardo Alberto Ríos-Osório ${ }^{1}$ y Clara Inés Nicholls-Estrada ${ }^{2}$

\section{RESUMEN}

El objetivo de este artículo fue caracterizar unidades familiares agroecológicas en el oeste de Santa Catarina, Brasil, con énfasis en la producción de leche. En el estudio se utilizaron herramientas etnográficas y se hizo un abordaje metodológico integrador con la aplicación de métodos de investigación descriptiva transversal por medio del uso de herramientas mixtas, en seis unidades familiares. Las principales características de las unidades se describieron mediante siete categorías de análisis, propuestas para representar los propósitos del proceso agroecológico, denominadas: seguridad alimentaria y nutricional, eficiencia productiva y energética, reproducción social de la familia, autonomía frente a los mercados, manejo ecológico y uso de tecnologías apropiadas localmente, autonomía y equidad en la toma de decisión y en la gestión del trabajo, y satisfacción. Los aspectos relacionados con la eficiencia productiva y energética de los sistemas productivos, la autonomía frente a los mercados y la reproducción social de la familia se identificaron como limitantes del proceso agroecológico en las unidades de análisis. El estudio generó reflexiones con las familias y destacó factores relevantes para la toma de decisiones hacia una mayor sostenibilidad y para futuros estudios de comprensión del proceso agroecológico.

Palabras clave: agricultura familiar, agroecología, sistemas complejos, indicadores.

\begin{abstract}
The aim of this paper was to characterize agroecological family units in the West of Santa Catarina/Brazil, with emphasis on milk production. Was using ethnographic tools and was do an integrative methodological approach with application of cross descriptive investigation methods using mixed tools, in six family units. The main characteristics of units were described using seven categories of analysis, proposals to represent the purposes of agroecological process, named: food and nutrition security, productive and energetic efficiency, social reproduction of the family, autonomy against markets, ecological management and use of locally appropriate technologies, autonomy and equity in the decision making and in the work management, and satisfaction. Aspects related to productive and energetic efficiency of the production systems, autonomy against markets and social reproduction of the family were identified as limiting of the agroecological process in the units of analysis. The study generated reflections with the families and highlighted relevant factors for decision making toward greater sustainability and for future studies to understanding the agroecological process.
\end{abstract}

Key words: family farming, agroecology, complex systems, indicators.

\section{Introducción}

En los últimos años la ganadería lechera ha aumentado la productividad por animal, así como también el hato lechero. Se estima que la producción mundial de leche pasará de 580 millones de toneladas en 1999/2001 a aproximadamente 1.000 millones de toneladas en 2050 (FAO, 2006). En Brasil, el número total de bovinos es de 211.764 mil millones de cabezas (23 millones son vacas en ordeño) y la región sur ostenta la mayor productividad por animal (2.674 litros/vaca/año), cifra que equivale

\footnotetext{
1 Universidad de Antioquia, Grupo de Investigación Salud y Sostenibilidad. Calle 67 \# 53-108, Ciudad Universitaria, bloque 5 , oficina 135, Medellín, Colombia. leonardo.rios@udea.edu.co

2 University of California, College of Nature Resources.

* Autor para correspondencia: anapaulalica@yahoo.com.br.
}

Fecha de Recepción: 29 Abril, 2016.

Fecha de Aceptación: 14 Noviembre, 2016.

DOI: $10.4067 /$ S0718-34292016005000031. 
al 34\% de la producción nacional pero con tan solo el 13\% de las cabezas del país (IBGE, 2014). De los tres estados del sur: Paraná, Santa Catarina y Rio Grande do Sul, en 2013 Santa Catarina fue el único que demostró un número creciente de cabezas, con un aumento de $27 \%$ en la cantidad de vacas ordeñadas en relación con el año anterior, además de una productividad de 2.577 litros/vaca/ año (IBGE, 2014).

Pese a la importancia de la actividad lechera en Santa Catarina poco se sabe acerca del estado de la sostenibilidad de las unidades productivas desde la perspectiva de sostenibilidad entendida como la resiliencia socioecológica de los sistemas complejos (Salas-Zapata et al., 2012). Precisamente, en este artículo se analizan fincas ganaderas en proceso agroecológico como unidades familiares, que son un medio de vida, tanto social como ecológico, para las familias en el espacio agrario donde se desarrollan procesos de resistencia y adaptación hacia la regenerabilidad y la mayor sostenibilidad; por tanto, existen otros procesos además del productivo que requieren ser integrados en la concepción de las unidades familiares de producción, asumidas de esta manera como sistemas socioecológicos agrarios complejos. El objetivo del estudio fue caracterizar de manera socioecológica seis unidades familiares con proceso agroecológico en el oeste de Santa Catarina, Brasil, con énfasis en la producción de leche. Aunque el artículo presenta en forma extractada la descripción de las unidades, uno de los intereses del mismo fue lograr una aproximación a la complejidad inherente a ellas y generar procesos reflexivos con las familias, los que son la base para la toma de decisiones y comprensión de los problemas de insostenibilidad.

\section{Materiales y Métodos}

En el estudio se utilizó la etnografía y se hizo un abordaje metodológico integrador con la aplicación de métodos de investigación descriptiva transversal por medio del uso de instrumentos mixtos (Hernández-Sampieri et al., 2010).

La propuesta se realizó en el sur de Brasil, en la región oeste del Departamento de Santa Catarina, zona rural de los municipios de Quilombo (Lat. $26^{\circ}: 43^{\prime}: 33^{\prime \prime}$ S; Long. 52 $: 43^{\prime}: 15^{\prime \prime}$ W; Alt. 425 msnm), Formosa do Sul (Lat. 26':38':49" S; Long. $52^{\circ}: 47^{\prime}: 38^{\prime \prime}$ W; Alt. $500 \mathrm{msnm}$ ) y Novo Horizonte

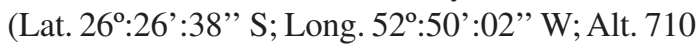

msnm). Con base en el Censo Demográfico (IBGE, 2010), la región está compuesta por 98 municipios con una población que alcanza los 1.20 .712 habitantes, con participación equitativa de hombres y mujeres; sin embargo, aproximadamente el $72 \%$ de la población es urbana y el $28 \%$ es rural. Aún según el Censo, la densidad poblacional es de 44 hab. $/ \mathrm{km}^{2}$ y los principales renglones de la economía están en los sectores de servicios, industria y agropecuaria. Los municipios en donde están las unidades presentan clima mesotérmico húmedo, sin estación seca, del tipo Cfa, es decir, subtropical (caliente y húmedo) con lluvias bien distribuidas en el verano (Köppen y Geiger, 1936).

Las familias seleccionadas estaban activas en la producción ecológica de leche de vaca en 2013 y cumplían con los siguientes criterios de inclusión: estar en el catastrado como productor orgánico en el Núcleo Noroeste Catarinense de Agroecología (RedeEcovida, 2013), y estar en el Catastro Nacional de Productores Orgánicos del Ministerio de Agricultura, Pecuaria y Abastecimiento de Brasil (MAPA, 2013). Se incluyeron 24 familias, de la que se excluyeron cinco por no presentar la leche como producto orgánico en los catastros. Luego, $30 \%$ de estas fueron seleccionadas por sorteo para conformar la muestra compuesta por seis unidades localizadas en los municipios de Quilombo (2), Novo Horizonte (3) y Formosa do Sul (1) en el oeste de Santa Catarina, Brasil. En el artículo las familias están identificadas con la letra " $F$ " adicionada a las iniciales de su primer apellido.

Inicialmente, se empleó un cuestionario sociodemográfico para la recolección de información acerca de la estructura familiar, la edad, el género, la escolaridad y cuestiones sobre el tamaño de la finca y el inicio del proceso agroecológico. Posteriormente, se hizo una sensibilización y un acercamiento a la percepción de los miembros de las familias sobre el significado de sostenibilidad mediante el uso de cartografía social. Luego, de manera colectiva, se construyeron parámetros de sostenibilidad, si bien algunos parámetros relevantes para la caracterización socioecológica fueron complementados y establecidos con base en la literatura.

Cada parámetro establecido fue inscrito en una de tres dimensiones: económica, cultural y ambiental, con el propósito de instigar a los sujetos para que mencionaran diferentes aspectos a partir de un concepto integral y global de la sostenibilidad y para que visualizaran el significado que tiene su posición en 
cuanto a los parámetros en cada dimensión. Además, los parámetros fueron ponderados según su importancia para las familias por medio de la asignación de una, dos o tres estrellas, considerando que una estrella indica el nivel más bajo de importancia y tres el más alto (Sarandón y Flores, 2009).

Todos los parámetros fueron usados como base para la construcción de indicadores con énfasis en el proceso agroecológico y en la producción de leche. Se establecieron los métodos de evaluación y los valores ideales para la realidad en estudio. Luego, los indicadores fueron evaluados con las familias y comparados con los valores ideales establecidos anteriormente. En secuencia, se empleó el "método del semáforo" (Cammaert et al., 2007) el que es una propuesta de interpretación en escala cualitativa basada en colores con el propósito de que los sujetos de estudio comprendan que significado tiene la evaluación de la situación de su finca. Para facilitar el análisis, todos los resultados de la evaluación, independientemente de su unidad de origen fueron estandarizados mediante una escala numérica que se corresponde con los colores del semáforo de la siguiente manera: 8 a 6 para situación deseable (verde), 5 a 3 para situación regular (amarillo) y 2 a 0 para situación indeseable (rojo).

Los datos fueron recolectados en cuestionarios, entrevistas semiestructuradas, relatos de experiencia y evaluaciones en campo. Los datos cualitativos fueron obtenidos de las grabaciones las que fueron transcritas, sistematizadas, codificadas, categorizadas y analizadas en el programa ATLAS TI 6.0. Los datos cuantitativos para la eficiencia productiva y energética se analizaron en el software libre "Energía 3.01" de Funes-Monzote et al. (2008), en tanto que los datos referentes al costo de producción de la leche se analizaron en las planillas de CONSELEITE (2013).

Se definieron siete categorías utilizadas para apoyar la descripción de las unidades familiares agroecológicas: seguridad alimentaria y nutricional, eficiencia productiva y energética, reproducción social de la familia, autonomía frente a los mercados, manejo ecológico y uso de tecnologías apropiadas localmente, autonomía y equidad en la toma de decisión y en la gestión del trabajo, y satisfacción. Por tanto, para la caracterización socioecológica de las unidades fue necesaria, además de la información secundaria y de los relatos de experiencia, la evaluación de 24 parámetros y 54 indicadores dentro de las categorías de análisis propuestas (Tabla 1).
Al final, para sintetizar los resultados encontrados con los indicadores se calcularon siete índices, uno por categoría, de acuerdo con la metodología descrita por Sarandón y Flores (2009), para entonces se calculó un Índice General Familiar (IGF) que es resultado del promedio de los siete índices.

\section{Resultados y Discusión}

La información sociodemográfica, evidencia que aproximadamente el $65 \%$ de la muestra son adultos con más de 30 años, $8 \%$ son jóvenes entre 15 y 20 años, y $27 \%$ son niños hasta de 10 años. También, mostró que el $50 \%$ de las familias son nucleadas y la otra mitad son extensas, con la presencia de los abuelos. Los datos indican que el promedio en cuanto a la cantidad de personas que conforman las familias, es de 4,3, con edad promedio de 34,4 años; además, la conformación es equitativa en proporción de género (50\% femenino y $50 \%$ masculino). Los datos sobre la escolaridad indicaron que tan solo el 23,5\% de los adultos terminaron los estudios básicos; sin embargo, todos los niños y jóvenes en edad escolar estaban en proceso normalizado. Como resultados generales se encontró que existe un déficit en el proceso formativo de los adultos y que la escolarización disminuyó a medida que se avanzó en los niveles educativos.

El proceso agroecológico en cada unidad de análisis empezó en diferentes momentos y por diferentes motivaciones; no obstante, hay un discurso predominante sobre la construcción de conciencia socioambiental y optimización de la salud (Tabla 2).

A seguir, de manera sucinta, se presenta un panorama general de cada unidad de análisis a partir de los índices calculados para cada una de las siete categorías propuestas (Tabla 3).

A continuación, se hace una descripción de los resultados más importantes a partir de cada categoría propuesta:

\section{Seguridad alimentaria y nutricional}

El concepto de seguridad alimentaria y nutricional, propuesto por el Consejo Nacional de Seguridad Alimentaria y Nutricional de Brasil y aprobado por el gobierno como ley (Brasil, 2006), define como un derecho de todos el acceso regular y permanente a suficiente cantidad y calidad de alimentos, respetando la diversidad cultural de los 


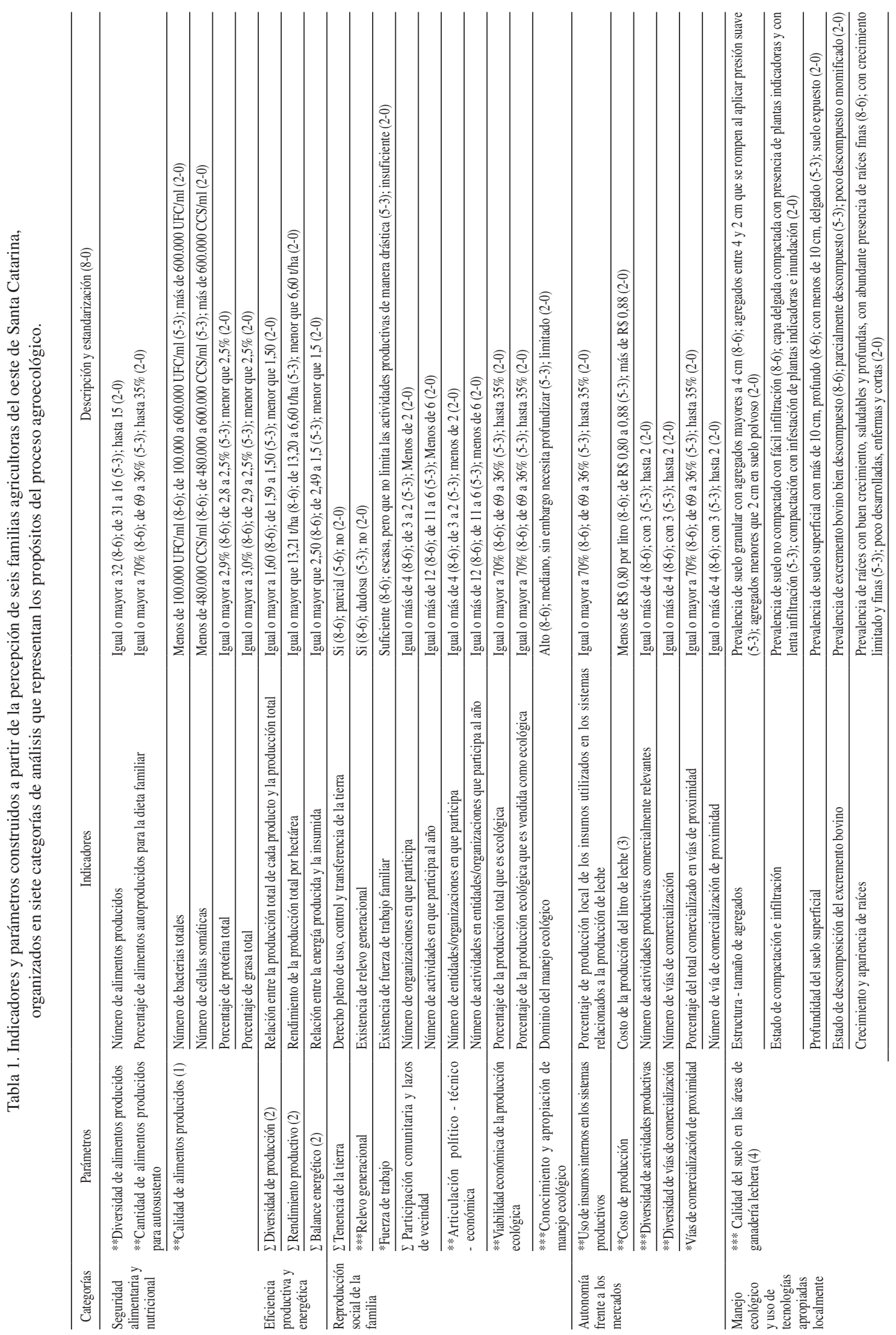




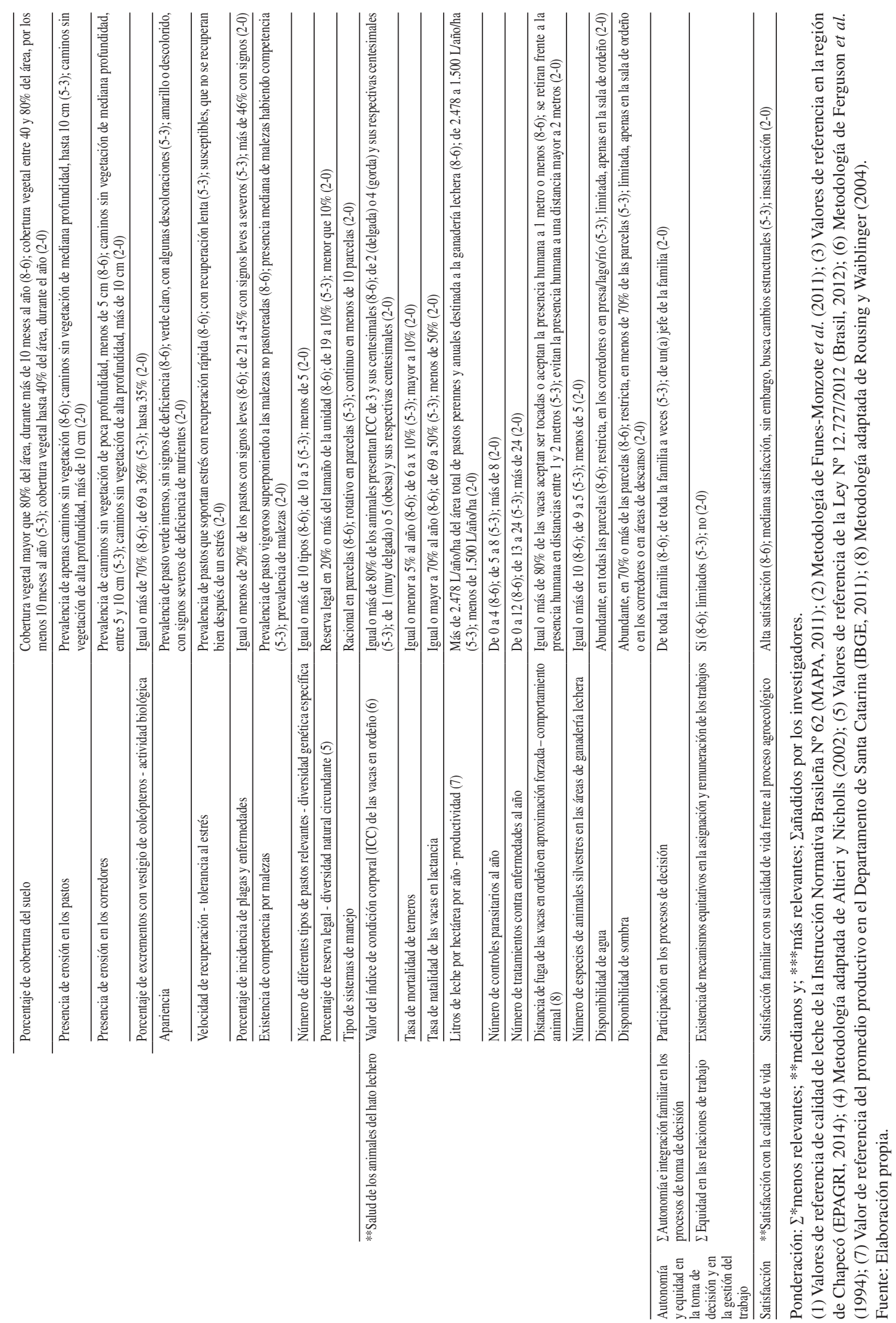


Tabla 2. Información básica de seis unidades familiares en proceso agroecológico en la región oeste de Santa Catarina, Brasil, en 2013.

\begin{tabular}{lccccl}
\hline Familia* & $\begin{array}{c}\text { Estructura } \\
\text { familiar }\end{array}$ & $\begin{array}{c}\text { Número } \\
\text { de } \\
\text { miembros }\end{array}$ & $\begin{array}{c}\text { Área de } \\
\text { la unidad } \\
\text { (ha) }\end{array}$ & $\begin{array}{c}\text { Inicio del proceso } \\
\text { agroecológico } \\
\text { (año) }\end{array}$ & $\begin{array}{c}\text { Principal motivación para } \\
\text { iniciar el proceso agroecológico } \\
\text { (testimonio) }\end{array}$ \\
\hline FGr & Extensa & 7 & 13,5 & 2007 & "Disminuir costos de producción" \\
FFo & Extensa & 6 & 20,4 & 2006 & "Productos mejores para el consumo familiar y la venta" \\
FCo & Nuclear & 3 & 4,3 & 1995 & "Conciencia y tradición familiar" \\
FGh & Nuclear & 3 & 46 & 2004 & "Conciencia social y ambiental" \\
FPi & Extensa & 3 & 24 & 2000 & "Conciencia ambiental y salud de la familia" \\
FMe & Nuclear & 4 & 19,3 & 2009 & "Salud de los animales, de la familia y de los consumidores"
\end{tabular}

* Las familias fueron identificadas con la tetra "F" adicionada a las iniciales de su apellido.

Fuente: Elaboración propia a partir de datos de campo (2013).

Tabla 3. Panorama general en escala (0-8) de las categorías representativas del proceso agroecológico, con énfasis en la producción de leche, en seis unidades familiares en la región oeste de Santa Catarina, Brasil, en 2013.

\begin{tabular}{|c|c|c|c|c|c|c|c|}
\hline Categoría/Familia* & FGr & FFo & FCo & FGh & $\mathrm{FPi}$ & $\mathrm{FMe}$ & Promedio \\
\hline Seguridad alimentaria y nutricional & 7,75 & 8,00 & 5,83 & 5,42 & 7,25 & 5,33 & 6,59 \\
\hline Eficiencia productiva y energética & 3,67 & 5,00 & 5,00 & 3,67 & 4,33 & 4,33 & 4,33 \\
\hline Reproducción social de la familia & 5,04 & 5,73 & 6,88 & 5,27 & 6,19 & 5,08 & 5,69 \\
\hline Autonomía frente a los mercados & 5,25 & 4,65 & 6,15 & 4,25 & 7,60 & 2,90 & 5,13 \\
\hline Manejo ecológico y uso de tecnologías apropiadas localmente & 6,31 & 6,37 & 6,26 & 6,44 & 6,01 & 6,68 & 6,34 \\
\hline Autonomía y equidad en la toma de decisión y en la gestión del trabajo & 7,50 & 7,50 & 7,50 & 4,50 & 7,50 & 6,00 & 6,75 \\
\hline Satisfacción & 7,00 & 6,00 & 8,00 & 3,00 & 6,00 & 6,00 & 6,00 \\
\hline Índice general familiar & 6,07 & 6,18 & 6,52 & 4,65 & 6,41 & 5,19 & 5,83 \\
\hline
\end{tabular}

*Las familias fueron identificadas con la tetra " $F$ " adicionada a las iniciales de su apellido.

Fuente: Elaboración propia a partir de datos de campo (2013).

pueblos. En esta categoría se obtuvo una situación favorable en tres de las unidades y regular en las tres restantes, pero estas últimas estaban muy cercanas al nivel deseable.

Solo una familia evidenció la máxima calificación en los seis indicadores evaluados; esta familia cosechó 60 alimentos diferentes, produjo el $85 \%$ de los alimentos autoconsumidos y presentó la mejor calidad de la leche producida. Sin embargo, las otras dos familias con desempeño deseable presentaron alto promedio anual de bacterias totales pero bajo conteo de células somáticas, resultados que indican problemas en la condición higiénico-sanitaria. Para Pinto et al. (2006), la alta contaminación bacteriana en la leche cruda refrigerada se asocia con una higiene inadecuada durante el ordeño, como uso de agua contaminada, equipos mal lavados, ambiente y animales en malas condiciones higiénicas, además de fallas en procedimientos de almacenamiento como el cálculo incorrecto de temperatura y tiempo de refrigeración.

\section{Eficiencia productiva y energética}

De acuerdo con la literatura científica los sistemas agrícolas, que tratan de maximizar el uso de la fuentes renovables de energía para lograr el incremento de la productividad, deberían lograr una alta productividad, equiparable a una alta eficiencia en el uso de la energía (Funes-Monzote et al., 2011). Sin embargo, en este estudio todas las unidades demostraron estar en una situación regular en esta categoría, con un promedio general de 4,33 en la escala de 0 a 8 .

En este estudio se encontró que las unidades con alta diversidad de producción no necesariamente presentan alta productividad y balance energético eficiente. Estos hallazgos coinciden con los del estudio de Funes-Monzote et al. (2011) realizado en Cuba. La unidad más productiva demostró muy baja diversidad de producción y un balance energético indeseable con alta importación de insumos externos, mientras que la unidad con 
mejor balance energético demostró el más bajo rendimiento y la menor diversidad productiva. Conviene resaltar que esta unidad posee un área verde grande y una intensidad de manejo baja, y como propone el autor puede mejorar su eficiencia por medio de la diversificación funcional por medio del diseño integrado de los sistemas productivos.

\section{Reproducción social de la familia}

La autonomía de la agricultura familiar campesina consiste en la reproducción social de la familia sustentada en la capacidad de suplir las necesidades inmediatas y futuras con centralidad en el patrimonio familiar (Wanderley, 2009). En este sentido, es evidente que todas las categorías propuestas en este trabajo influyen sobre la reproducción social en el campo; sin embargo, se extrajeron los aspectos más determinantes del proceso. Esta categoría presentó situación favorable en dos unidades y regular en las otras cuatro, pero todas con valoraciones muy cercanas.

Los indicadores con más familias en situación desfavorable fueron el relevo generacional, la articulación político-económica, la viabilidad económica de la producción ecológica (comercialización) y el conocimiento y apropiación del manejo ecológico (capacidad técnico-productiva). Se observó que la familia con más tiempo en el proceso agroecológico obtuvo el mejor promedio ponderado de los indicadores, e incluso demostró, entre todas las familias, la más alta probabilidad de relevo generacional y una muy alta viabilidad económica de la producción ecológica, pues el $90 \%$ de todo lo que produjo es ecológico y de esta producción logró vender el 70\% como ecológica. Se observó que solamente las dos unidades con más tiempo en el proceso agroecológico lograron vender sus productos en el mercado como ecológicos, demostrando problemas en el proceso de comercialización en gran parte de las familias.

\section{Autonomía frente a los mercados}

$\mathrm{El}$ agricultor familiar campesino orienta sus acciones económicas hacia la autonomía; sin embargo, este no puede ser considerado como un actor independiente o aislado de la sociedad que lo circunda, pero, sí se refiere a una actitud de valoración de sus propios recursos y de creación de condiciones internas que garanticen la reproducción de su unidad familiar de producción y vida (Wanderley, 2009). En este sentido, en la categoría se evaluaron seis indicadores, en los que dos familias demostraron situación deseable, tres regular y una indeseable.

En la evaluación, una vez más, las dos familias con mayor tiempo en el proceso agroecológico demostraron el mejor desempeño, incluso con la comercialización de un alto volumen de productos en diferentes vías de proximidad, además de gran eficiencia económica en la producción lechera con bajo costo. La familia con menos tiempo en el proceso agroecológico presentó una situación de alta vulnerabilidad frente a los mercados, a pesar de tener un bajo costo de producción de la leche. Esta familia utiliza alta cantidad de insumos de la industria agropecuaria, comercialmente produce exclusivamente leche vacuna y vende para una única industria láctea, hecho que la ubica en alto riesgo económico. Además, la falta de diversificación de actividades económica refleja mejores niveles de autonomía y flexibilidad de los agricultores en la conducción de las relaciones de mercado, situación identificada en esta familia, que adicionalmente demostró el peor desempeño en seguridad alimentaria y nutricional.

\section{Manejo ecológico y uso de tecnologías apropiadas localmente}

La aplicación de principios, manejos y tecnologías en el proceso agroecológico ha desarrollado sistemas de producción con interacciones ecológicas que posiblemente explican el mejor comportamiento en dichos sistemas (Altieri y Nicholls, 2002). La categoría englobó indicadores de calidad del suelo, salud de los cultivos y del pasto, y salud de los animales. Las familias presentaron un promedio ponderado general de 6,34 en esta categoría de análisis, con lo que se demostró un manejo ecológico adecuado que es susceptible de mejoras al implementar prácticas ecológicas hacia una mayor salud del suelo, los cultivos y los animales. Al contrario de lo esperado, las familias con mejor desempeño en esta categoría fueron las dos con el IGF más bajo, con lo que quedó claro que el aspecto ecológico es necesario pero no garantiza un proceso agroecológico equitativo hacia la sostenibilidad socioecológica; las dos familias que llevan más tiempo en el proceso agroecológico, tuvieron el desempeño más bajo en esta categoría pero aun así obtuvieron los dos mejores IGF. 


\section{Autonomía y equidad en la toma de decisión y en la gestión del trabajo}

La sociedad humana debe fomentar la universalización de los valores del cuidado y la empatía hacia los humanos y la naturaleza; es así que, en ámbito de lo cotidiano, se dan virtudes y obligaciones morales relacionadas con la equidad y la sostenibilidad (Puleo, 2008). En las unidades familiares de producción y vida en proceso agroecológico, la estructura de poder debe ser esencialmente horizontal. Esta categoría refleja aspectos relacionados con la integración en los procesos de toma de decisión y la equidad en las relaciones de trabajo, en lo que cinco familias demostraron percepción positiva en relación con su realidad, y solo una familia valoró como baja la integración y la equidad en la vida familiar y de trabajo, pues había sobrecarga femenina (madre) en los trabajos productivos porque el padre, con estudio universitario completo, era articulador del proceso agroecológico en la región.

\section{Satisfacción}

Esta tiene que ver con suplir las necesidades humanas fundamentales, en este sentido, el estado de satisfacción en vivir hace referencia a la calidad de vida (D'Agostini y Fantini, 2008). La pregunta por la satisfacción en este estudio surge de la necesidad de identificar la percepción de las familias en cuanto a la calidad de su vida frente a las dinámicas socioecológicas que el proceso agroecológico proporciona; por tanto, la satisfacción se asocia de manera directa con el desempeño en las otras categorías. De las seis familias, cinco demostraron estar satisfechas. Nuevamente, la familia que lleva más tiempo en el proceso agroecológico, demostró la valoración más alta y, la familia con regular situación de equidad en la categoría anterior, valoró como regular su satisfacción, percibiendo la necesidad de cambios, lo que refleja su bajo desempeño en el IGF.

\section{Conclusiones}

El principal aporte de este estudio se centra en llevar todo el proceso investigativo con la participación activa de los sujetos de estudio, de manera consensual, para describir las unidades y generar procesos de reflexión familiar sobre su realidad socioecológica y posibilidades futuras hacia una mayor sostenibilidad. En este sentido, el estudio generó reflexiones con las familias y destacó factores relevantes para la toma de decisiones hacia una mayor sostenibilidad y para futuros estudios de comprensión del proceso agroecológico.

La agroecología en las unidades familiares con producción de leche es un proceso con potencial para ofrecer condiciones para una mayor sostenibilidad socioecológica en la región oeste de Santa Catarina; sin embargo, los aspectos relacionados con la eficiencia productiva y energética de los sistemas productivos, la autonomía frente a los mercados y la reproducción social de la familia fueron identificados como la limitante del proceso, demostrándose así debilidad técnico-productiva y problemas de comercialización de los productos ecológicos.

Las familias con más tiempo en el proceso agroecológico demostraron mejor desempeño general; sin embargo, la limitación de estas familias en relación a la capacidad técnica fue un hallazgo que puede ser explicado por los procesos de construcción del conocimiento y de desarrollo de habilidades innovadoras. Las familias que llevan menos tiempo en el proceso agroecológico demostraron mayor capacidad de experimentación para el manejo ecológico y el uso de tecnologías apropiadas localmente. Se observó que existen factores externos a las unidades familiares que limitan el desarrollo de innovaciones en el proceso agroecológico, quizá las dificultades encontradas durante el largo tiempo de resistencia de las familias más antiguas en el proceso y la falta de apoyo han dificultado la continuidad en la producción de novedades tecnológicas para un apropiado manejo ecológico. Son necesarios diseños integrados de los sistemas productivos mediante una diversificación funcional hacia una mayor eficiencia productiva así como energética. Por lo tanto, aún existe la necesidad de un nivel mayor de innovación con el fin de crear alternativas productivas concretas así como redes comerciales que estimulen al proceso agroecológico y que viabilicen la producción de leche ecológica con la agencia de las familias.

\section{Agradecimientos}

Este trabajo se realizó con el apoyo del Consejo Nacional de Desarrollo Científico y Tecnológico $\mathrm{CNPq} /$ Brasil. 


\section{Literatura Citada}

Altieri, M.; Nicholls, C.

2002. Un método agroecológico rápido para la evaluación de la sostenibilidad de cafetales. Manejo Integrado de Plagas y Agroecología 64: 17-24.

Brasil

2012. Lei $N^{o} 12.727$, de 17 de outubro de 2012. Diário Oficial da União de 18/10/2012. Brasília, Brasil. Disponible en: http://www.jusbrasil.com.br/diarios/41537746/dou-secao1-18-10-2012-pg-1 Consultado: 20/ene/2016. Brasil

2006. Lei $N^{o} 11.346$, de 15 de setembro de 2006. Diário Oficial da União de 18/09/06. Brasília, Brasil. Disponible en: http://www.jusbrasil.com.br/diarios/782516/pg1-secao-1-diario-oficial-da-uniao-dou-de-18-09-2006 Consultado: 20/ene/2016.

Cammaert, C.; Palacios, M.T.; Arango, H.; Calle, Z.

2007. Mi finca biodiversa: herramienta didáctica para la planificación de la biodiversidad en la finca. $1^{\text {a }}$ ed. Instituto Alexander von Humboldt. Bogotá, Colombia, 55 p.

CONSELEITE - Conselho Paritário de Produtores Rurais e

Indústrias de Leite do Estado de Santa Catarina

2013. Planilhas de custos de produção do leite. Brasil: Epagri. Disponible en: http://www.epagri.sc.gov.br/?page_id=2696 Consultado: 10/ene/2015.

D’Agostini, L.R.; Fantini, A. C.

2008. Quality of life and quality of living conditions in rural areas: distinctively perceived and quantitatively distinguished. Social Indicators Research 89 (3): 487-499.

EPAGRI - Empresa de Pesquisa Agropecuária e Extensão Rural de Santa Catarina

2014. Sintese anual da agricultura de Santa Catarina 20132014. Brasil: Epagri. Disponible en: http://docweb.epagri. sc.gov.br/website_cepa/publicacoes/Sintese_2014.pdf Consultado:10/ene/2016.

FAO - The Food and Agriculture Organization of the United Nations

2006. World Agriculture: towards 2030/2050. Interim report. Agricultural Development Economics Division. FAO. Rome, Italy, $71 \mathrm{p}$.

Ferguson, J.D.; Galligan, D.T.; Thomsen, N.

1994. Principal descriptors of body condition score in Holstein cows. Journal of Dairy Science 77: 2695-2703.

Funes-Monzote, F.; Castro, J.; Rodrígues, N. V.; Gonçalves, A.L.; Costa, D.P.; Álvarez, Y.R.

2008. Software Energía: balance energético de agroecosistemas (versión 3.01). Cuba.

Funes-Monzote, F.R.; Martín, G.J.; Suárez, J.; Blanco, D.; Reyes, F.; Cepero, L., Rivero, J.L; Rodríguez, E.; Savran, Valentina; del Valle, Yadiris; Cala, Marlenis; Vigil, María del C.; Sotolongo, J.A.; Boillat, S.; Sánchez, J.E.

2011. Evaluación inicial de sistemas integrados para la producción de alimentos y energía en Cuba. Pastos y Forrajes 34 (4): 445-462.

Hernández-Sampieri, R.; Fernández-Collado, C.; Baptista-Lucio, P.

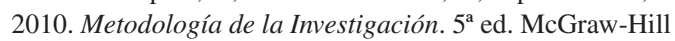
Interamericana Editores S.A. Lima, Perú, 613 p.
IBGE - Instituto Brasileiro de Geografia e Estatística

2014. Produção da pecuária municipal: 2013. V. 41. Rio de Janeiro: IBGE. Disponible en: http://biblioteca.ibge. gov.br/visualizacao/periodicos/84/ppm_2013_v41_br.pdf Consultado: 10/mar/2015.

IBGE - Instituto Brasileiro de Geografia e Estatística

2011. Produção da pecuária municipal: 2011. V. 39. Rio de Janeiro: IBGE. Disponible en: http://ftp.ibge.gov.br/ Producao_Pecuaria/Producao_da_Pecuaria_Municipal/2011/ ppm2011.pdf Consultado: 10/mar/2015.

IBGE - Instituto Brasileiro de Geografia e Estatística

2010. Censo demográfico 2010. Brasil: IBGE. Disponible en: http://cidades.ibge. gov.br/xtras/perfil.php?codmun=421690 Consultado: 10/mar/2015.

Köppen, G.; Geiger. M.

1936. Das geographische System der Klimate, Handbuch der Klimatologie [The Geographical System of the Climate, Handbook of Climatology]. Band I, Teil C [Vol. 1, Part C]. Verlag von Gebrüder Borntraeger. Berlin, Alemania. 44 p. MAPA - Ministério da Agricultura, Pecuária e Abastecimento 2013. Cadastro nacional dos produtores orgânicos. Brasil: MAPA. Disponible en: http://www.agricultura.gov.br/ desenvolvimento-sustentavel/organicos/cadastro-nacional. Consultado: 01/ene/2013.

MAPA - Ministério da Agricultura, Pecuária e Abastecimento 2011. Instrução Normativa $N^{o} 62$, de 29 de dezembro de 2011. Diário Oficial da União de 30/12/2011. Brasil: MAPA. Disponible en: http://www.jusbrasil.com.br/diarios/33395065/ dou-secao-1-30-12-2011-pg-6 Consultado: 10/ene/2015.

Pinto, C.L. de O.; Martins, M.L.; Vanetti, M.C.D.

2006. Qualidade microbiológica de leite cru refrigerado e isolamento de bactérias psicrotróficas proteolíticas. Ciência e Tecnologia de Alimentos 26 (3): 645-651.

Puleo, A.H.

2008. Libertad, igualdad, sostenibilidad. Por un ecofeminismo ilustrado. ISEGORÍA: Revista de Filosofía Moral y Política 38: 39-59.

RedeEcovida

2013. Documento de aprovação ou de renovação da conformidade Orgânica. Formosa do Sul: Núcleo Noroeste Catarinense de Agroecologia.

Rousing, T.; Waiblinger, $\mathrm{S}$.

2004. Evaluation of on-farm methods for testing the humananimal relationship in dairy herds with cubicle loose housing systems-test-retest and inter-observer reliability and consistency to familiarity of test person. Applied Animal Behaviour Science 85: 215-231.

Salas-Zapata, W. A.; Ríos-Osorio, L.A. y Álvarez-Del-Castillo, J. 2012. Marco conceptual para entender la sustentabilidad de los sistemas socioecológicos. Ecología Austral 22: 74-79.

Sarandón, S.J.; Flores, C.C.

2009. Evaluación de la Sustentabilidad en Agroecosistemas: una propuesta metodológica. Revista Agroecología 4: 19-28.

Wanderley, M. de N.B.

2009. O Mundo Rural como um Espaço de Vida: reflexões sobre a propriedade da terra, agricultura familiar e ruralidade. $1^{\text {a }}$ ed. Editora da UFRGS. Porto Alegre, Brasil, 328 p. 
